\title{
Number of Children in Household
}

National Cancer Institute

\section{Source}

National Cancer Institute. Number of Children in Household. NCI Thesaurus. Code C19309.

a demographics parameter useful for identifying socio-economic groups. 